\title{
Material constant sensitivity boundary integral equation for anisotropic solids
}

\author{
Rafael Gallego*, ${ }^{*}$, Lucia Comino and Alberto Ruiz-Cabello \\ Department of Structural Mechanics, University of Granada, Spain
}

\begin{abstract}
SUMMARY
In this paper, the material constant sensitivity boundary integral equation is presented, and its numerical solution proposed, based on boundary element techniques. The formulation deals with plane problems with general rectilinear anisotropy. Expressions for the computation of sensitivities for displacements, tractions, strains and stresses are derived, both for boundary and interior points. The sensitivities can be computed with respect to the bulk material properties or to the properties of part of the domain (inclusions, coatings, etc.). To assess the accuracy of the proposed approach, the computed results are compared to analytical ones derived from exact solutions obtained by complex potential theory, when possible, or finite difference derivatives otherwise. Copyright (C) 2005 John Wiley \& Sons, Ltd.
\end{abstract}

KEY WORDS: material property sensitivity; two-dimensional anisotropic elasticity; inverse problem; boundary element method; boundary integral equations

\section{INTRODUCTION}

The identification of material properties of structural or mechanical elements is an active research area, due to the importance of this topic within the framework of maintenance and monitoring of high-performance structural systems. Non-isotropic materials are widely used in these kind of systems, and it is therefore of great interest to be able to predict the integrity and reliability of these materials. Since the elastic properties of the material can be affected by several factors during the life-time of the structure, their monitoring is indispensable to assure the safety and serviceability of the system. However, tests designed for laboratory conditions for evaluation of material properties are seldom suitable for an operating structure, since they usually entail the use of standardized probes and loading conditions, which cannot be reproduced in real structures. Examples of these applications are the monitoring of composite wings in

*Correspondence to: Rafael Gallego, Department of Structural Mechanics, Universidad de Granada, Ed. Politécnico, Avda Fuentenueva s/n, 18002 Granada, Spain.

†E-mail: gallego@ugr.es 
aeronautical applications, and the monitoring and maintenance of composite reinforcements in civil engineering structures $[1,2]$.

Non-destructive techniques for identifying material properties of structural elements combine partial experimental measurements with full numerical models of the system [3]. The parameters to be identified are obtained by the solution of an inverse problem, minimizing the discrepancy between the measured response and the computed one $[4,5]$.

While the literature is extensive regarding the identification of material constant for isotropic materials (see References [6-11]), the non-isotropic case has received much less attention. For this problem, some of the approaches deal with plate models, using either static or modal measurements [12-15], but very few works employ a two- or three-dimensional elastic model of the mechanical or structural element to solve the identification problem. Huang et al. [16] identify orthotropic elastic constants using a two-dimensional BEM model and static measurements. Heylinger et al. [17] combine resonant ultrasound spectroscopy (natural frequencies) with the Ritz method to obtain the material constants of general anisotropic materials, but due to the limitations of their numerical modelling, only simple domains (parallelepipeds) can be considered.

For the modelling, numerical methods have to be used to deal with complex geometries or/and loading conditions. Although the finite element method (FEM) may be advantageous when dealing with bulk or non-linear material properties, for specimens under elastic loadingswhich is the case in most of the non-destructive techniques-the boundary element method (BEM, Reference [18]) can be more accurate and computationally efficient than other popular techniques. Moreover, when dealing with the identification of both the properties and shape of an unknown domain (for instance, an inclusion) the BEM becomes clearly advantageous [11].

Regardless of the numerical modelling employed and the minimization approach for the solution of the inverse problem, an issue of the foremost importance is the computation of the gradient of the solution provided by the model with respect to the material constant. Although finite differences have been widely used, the computational cost incurred is high and inaccuracies in the derivative calculation may lead to unstable algorithms due to the ill-posedness of inverse problems.

In this paper, we contribute to the topic of material constant identification by developing a boundary integral equation (BIE) for the computation of displacement, traction, strain and stress derivatives with respect to the material constant of a two-dimensional rectilinearly anisotropic material. A numerical procedure based on standard boundary element techniques is developed. The singularity order of the new kernels is assessed and the integration procedures presented. A series of applications is reported where the results are compared with analytically computed ones, when available, including piece-wise heterogeneous cases.

\section{BOUNDARY ELEMENT METHOD FOR ANISOTROPIC MATERIALS}

The analytical solutions of anisotropic elastic problems can be found for a limited number of cases, only when the geometry and loading conditions are very simple. To solve more realistic problems one needs to resort to numerical approximate approaches. BEM is a collocation method which allows their numerical resolution, and it is based on the boundary integral representation of the displacements. This is a well-known technique for anisotropic elastic solids (see the books [19-21] and references therein). In this section, the main formulae for 
the method are briefly reviewed, in order to provide the minimum background for the next sections.

The well-known BIE which governs the elastic displacement field is given by

$$
c_{i j}(\mathbf{y}) u_{j}(\mathbf{y})+f_{\Gamma} T_{i j}(\mathbf{z}, \mathbf{y}) u_{j}(\mathbf{z}) \mathrm{d} \Gamma=\int_{\Gamma} U_{i j}(\mathbf{z}, \mathbf{y}) t_{j}(\mathbf{z}) \mathrm{d} \Gamma
$$

being $c_{i j}(\mathbf{y})$ the free term, which depends on the location of the collocation point $\mathbf{y} \in \Gamma$ [18] where $\Gamma$ is the boundary of the domain $\Omega$. The free term is $c_{i j}=\frac{1}{2} \delta_{i j}$ at smooth boundary points. Zero body forces have been assumed.

The kernels $T_{i j}$ and $U_{i j}$ are the anisotropic displacement and stress fundamental solutions, which are obtained using a complex potential representation $[19,22]$. Their expression is,

$$
\begin{aligned}
U_{i j}(\mathbf{z}, \tilde{\mathbf{z}}) & =2 \mathbb{R e}\left[p_{j 1} A_{i 1} \ln \left(z_{1}-\tilde{z}_{1}\right)+p_{j 2} A_{i 2} \ln \left(z_{2}-\tilde{z}_{2}\right)\right] \\
T_{i j}(\mathbf{z}, \tilde{\mathbf{z}}) & =2 \mathbb{R e}\left[\frac{q_{j 1} A_{i 1}}{z_{1}-\tilde{z}_{1}}\left(\mu_{1} n_{1}-n_{2}\right)+\frac{q_{j 2} A_{i 2}}{z_{2}-\tilde{z}_{2}}\left(\mu_{2} n_{1}-n_{2}\right)\right]
\end{aligned}
$$

where $\mu_{s}$ are two pairs of conjugate roots of the material's characteristic polynomial,

$$
\beta_{11} \mu^{4}-2 \beta_{16} \mu^{3}+\left(\beta_{12}+\beta_{66}\right) \mu^{2}-2 \beta_{16} \mu+\beta_{22}=0
$$

$q_{1 s}=\mu_{s}, q_{2 s}=-1 ; n_{i}$ are the components of the outward normal to the boundary; $p_{i k}$ is given by the equations

$$
\begin{aligned}
& p_{1 k}=\beta_{11} \mu_{k}^{2}+\beta_{12}-\beta_{16} \mu_{k} \\
& p_{2 k}=\beta_{12} \mu_{k}+\frac{\beta_{22}}{\mu_{k}}-\beta_{26}
\end{aligned}
$$

( $\beta_{i j}$ the reduced elastic constant), and $A_{i s}$ are complex constants which are computed solving the following system of equations:

$$
\left(\begin{array}{cccc}
1 & -1 & 1 & -1 \\
\mu_{1} & -\bar{\mu}_{1} & \mu_{2} & -\bar{\mu}_{2} \\
p_{11} & -\bar{p}_{11} & p_{12} & -\bar{p}_{12} \\
p_{21} & -\bar{p}_{21} & p_{22} & -\bar{p}_{22}
\end{array}\right)\left(\begin{array}{c}
A_{i 1} \\
\bar{A}_{i 1} \\
A_{i 2} \\
\bar{A}_{i 2}
\end{array}\right)=\left(\begin{array}{c}
\frac{\delta_{i 2}}{2 \pi \imath} \\
\frac{\delta_{i 1}}{2 \pi \imath} \\
0 \\
0
\end{array}\right)
$$

where $l=\sqrt{-1}$ is the imaginary unit.

Applying standard boundary element discretization techniques to Equation (1), an algebraic system of equations is obtained,

$$
\mathbf{H u}=\mathbf{G t}
$$

where $\mathbf{u}$ and $\mathbf{t}$ are vectors which collect the displacements and tractions for all interpolation nodes on the boundary. After applying boundary conditions and re-arranging the system of equations 
one gets,

$$
\mathbf{A x}=\mathbf{f}
$$

whose solution yields the unknown displacements and tractions on the boundary.

\subsection{Computation of strains and stresses at interior and boundary points}

Differentiating Equation (1) with respect to the co-ordinates of a collocation point $\tilde{\mathbf{z}} \in \Omega \backslash \Gamma$ one attains the integral equation for the strains,

$$
\varepsilon_{i k}(\tilde{\mathbf{z}})=\int_{\Gamma}\left[V_{i j k}(\mathbf{z}, \tilde{\mathbf{z}}) t_{j}(\mathbf{z})-S_{i j k}(\mathbf{z}, \tilde{\mathbf{z}}) u_{j}(\mathbf{z})\right] \mathrm{d} \Gamma
$$

where the kernels are,

$$
\begin{aligned}
& V_{i j k}(\mathbf{z}, \tilde{\mathbf{z}})=\frac{1}{2}\left[\frac{\partial U_{i j}}{\partial z_{k}^{\prime}}+\frac{\partial U_{k j}}{\partial z_{i}^{\prime}}\right]=-\sum_{s=1}^{2} \mathbb{R e}\left[\frac{p_{j s} D_{i k s}}{z_{s}-z_{s}^{\prime}}\right] \\
& S_{i j k}(\mathbf{z}, \tilde{\mathbf{z}})=\frac{1}{2}\left[\frac{\partial T_{i j}}{\partial z_{k}^{\prime}}+\frac{\partial T_{k j}}{\partial z_{i}^{\prime}}\right]=\sum_{s=1}^{2} \mathbb{R e}\left[q_{j s} D_{i k s} \frac{\mu_{s} n_{1}-n_{2}}{\left(z_{s}-z_{s}^{\prime}\right)^{2}}\right]
\end{aligned}
$$

with $D_{i k s}=A_{i(s)} R_{k(s)}+A_{k(s)} R_{i(s)}$ (no sum in $\left.s\right) ; R_{1(s)}=1$ and $R_{2(s)}=\mu_{s}$.

The integral equation above is discretized using the same procedure as for the displacement BIE. Stresses at interior point can be obtained straightforwardly from the strains by application of Hooke's law.

Finally, strains and stresses at boundary points are computed from the boundary solution, combining the displacement derivative along the boundary computed by shape function differentiation and the components of the traction vector.

\section{MATERIAL SENSITIVITY BOUNDARY INTEGRAL EQUATION}

This study aims to obtain sensitivity expressions for displacements, tractions, strains and stresses with respect to variations in the elastic constants. The process starts with the differentiation of the boundary integral equation (1) with respect to an arbitrary compliance coefficient $a_{p q}$, leading to

$$
\begin{gathered}
\frac{1}{2} \delta u_{i}(\mathbf{y})+f_{\Gamma}\left(\frac{\partial T_{i j}(\mathbf{z}, \mathbf{y})}{\partial a_{p q}} u_{j}(\mathbf{z})+T_{i j}(\mathbf{z}, \mathbf{y}) \delta u_{j}(\mathbf{z})\right) \mathrm{d} \Gamma \\
=\int_{\Gamma}\left(\frac{\partial U_{i j}(\mathbf{z}, \mathbf{y})}{\partial a_{p q}} t_{j}(\mathbf{z})+U_{i j}(\mathbf{z}, \mathbf{y}) \delta t_{j}(\mathbf{z})\right) \mathrm{d} \Gamma
\end{gathered}
$$

where

$$
\delta u_{i}=\frac{\partial u_{j}}{\partial a_{p q}}, \quad \delta t_{i}=\frac{\partial t_{i}}{\partial a_{p q}}
$$


are the boundary displacement and traction sensitivities with respect to the arbitrary compliance constant, respectively.

In this equation, besides the standard nuclei $U_{i j}$ and $T_{i j}$, two new kernels arise. The computation of the derivatives of the fundamental solution is simple, although cumbersome.

\subsection{Kernel derivatives}

The derivatives of the kernels $U_{i j}(\mathbf{z}, \mathbf{y})$ and $T_{i j}(\mathbf{z}, \mathbf{y})$ are obtained by differentiation of Equations (2) and (3)

$$
\begin{aligned}
& \frac{\partial U_{i j}(\mathbf{z}, \mathbf{y})}{\partial a_{p q}} \\
& =\sum_{s=1}^{2} 2 \mathbb{R e}\left[\left(\frac{\partial p_{j s}}{\partial a_{p q}} A_{i s}+p_{j s} \frac{\partial A_{i s}}{\partial a_{p q}}\right) \ln \left(z_{s}-y_{s}\right)+p_{j s} A_{i s} \frac{\partial}{\partial a_{p q}} \ln \left(z_{s}-y_{s}\right)\right] \\
& \frac{\partial T_{i j}(\mathbf{z}, \mathbf{y})}{\partial a_{p q}} \\
& =\sum_{s=1}^{2} 2 \mathbb{R e}\left[\left(\frac{\partial q_{j s}}{\partial a_{p q}} A_{i s}+q_{j s} \frac{\partial A_{i s}}{\partial a_{p q}}\right) \frac{\mu_{s} n_{1}-n_{2}}{z_{s}-y_{s}}\right. \\
& \left.\quad+q_{j s} A_{i s}\left\{\frac{\partial}{\partial a_{p q}}\left(\frac{1}{z_{s}-y_{s}}\right)\left(\mu_{s} n_{1}-n_{2}\right)+\frac{1}{z_{s}-y_{s}}\left(\frac{\partial \mu_{s}}{\partial a_{p q}} n_{1}-n_{2}\right)\right\}\right]
\end{aligned}
$$

After differentiation with respect to the compliance coefficients, the new terms which appear in (15) can be calculated applying the chain rule,

$$
\frac{\partial}{\partial a_{p q}}\left(\frac{1}{z_{s}-y_{s}}\right)=-\frac{\partial \mu_{s}}{\partial a_{p q}}\left[\frac{x_{2}-x_{2}^{l}}{\left(z_{s}-y_{s}\right)^{2}}\right], \quad \frac{\partial}{\partial a_{p q}} \ln \left(z_{s}-y_{s}\right)=\frac{\partial \mu_{s}}{\partial a_{p q}} \frac{x_{2}-x_{2}^{l}}{z_{s}-y_{s}}
$$

Taking into account the definition of $p_{j k}$ and $q_{j k}$ (5), their derivatives are

$$
\frac{\partial q_{1 s}}{\partial a_{p q}}=\frac{\partial \mu_{s}}{\partial a_{p q}}, \quad \frac{\partial q_{2 s}}{\partial a_{p q}}=0, \quad \frac{\partial p_{j k}}{\partial a_{p q}}=\frac{\partial p_{j k}}{\partial \beta_{m n}} \frac{\partial \beta_{m n}}{\partial a_{p q}}
$$

where

$$
\left(\begin{array}{c}
\frac{\partial p_{1 s}}{\partial \beta_{m n}} \\
\frac{\partial p_{2 s}}{\partial \beta_{m n}}
\end{array}\right)=\left(\begin{array}{c}
\frac{\partial \beta_{11}}{\partial \beta_{m n}} \mu_{s}^{2}+2 \beta_{11} \mu_{s} \frac{\partial \mu_{s}}{\partial \beta_{m n}}+\frac{\partial \beta_{12}}{\partial \beta_{m n}}-\frac{\partial \beta_{16}}{\partial \beta_{m n}} \mu_{s}-\beta_{16} \frac{\partial \mu_{s}}{\partial \beta_{m n}} \\
\frac{\partial \beta_{12}}{\partial \beta_{m n}} \mu_{s}+\beta_{12} \frac{\partial \mu_{s}}{\partial \beta_{m n}}+\frac{1}{\mu_{s}} \frac{\partial \beta_{22}}{\partial \beta_{m n}}-\frac{\beta_{22}}{\mu_{s}^{2}} \frac{\partial \mu_{s}}{\partial \beta_{m n}}-\frac{\partial \beta_{26}}{\partial \beta_{m n}}
\end{array}\right)
$$

and $\partial \beta_{i j} / \partial \beta_{m n}=\delta_{i m} \delta_{j n}$.

The derivatives of the constants $A_{i s}$ are computed by solving a new system of equations that arises from the differentiation of the original one given in Equation (6)

$$
\mathbf{M} \boldsymbol{\delta} \mathbf{A}=-\boldsymbol{\delta} \mathbf{M A}
$$


where

$$
\boldsymbol{\delta} \mathbf{A}=\left(\begin{array}{l}
\frac{\partial A_{i 1}}{\partial a_{p q}} \\
\frac{\partial \bar{A}_{i 1}}{\partial a_{p q}} \\
\frac{\partial A_{i 2}}{\partial a_{p q}} \\
\frac{\partial \bar{A}_{i 2}}{\partial a_{p q}}
\end{array}\right), \quad \boldsymbol{\delta M}=\left(\begin{array}{cccc}
0 & 0 & 0 & 0 \\
\frac{\partial \mu_{1}}{\partial a_{p q}} & -\frac{\partial \bar{\mu}_{1}}{\partial a_{p q}} & \frac{\partial \mu_{2}}{\partial a_{p q}} & -\frac{\partial \bar{\mu}_{2}}{\partial a_{p q}} \\
\frac{\partial p_{11}}{\partial a_{p q}} & -\frac{\partial \bar{p}_{11}}{\partial a_{p q}} & \frac{\partial p_{12}}{\partial a_{p q}} & -\frac{\partial \bar{p}_{12}}{\partial a_{p q}} \\
\frac{\partial p_{21}}{\partial a_{p q}} & -\frac{\partial \bar{p}_{21}}{\partial a_{p q}} & \frac{\partial p_{22}}{\partial a_{p q}} & -\frac{\partial \bar{p}_{22}}{\partial a_{p q}}
\end{array}\right)
$$

To obtain the derivatives of the characteristic roots, polynomial (4) is differentiated, leading to

$$
\frac{\partial \mu_{k}}{\partial \beta_{m n}}=-\frac{\frac{\partial \beta_{11}}{\partial \beta_{m n}} \mu_{k}^{4}-2 \frac{\partial \beta_{16}}{\partial \beta_{m n}} \mu_{k}^{3}+\left(2 \frac{\partial \beta_{12}}{\partial \beta_{m n}}+\frac{\partial \beta_{66}}{\partial \beta_{m n}}\right) \mu_{k}^{2}-2 \frac{\partial \beta_{26}}{\partial \beta_{m n}} \mu_{k}+\frac{\partial \beta_{22}}{\partial \beta_{m n}}}{4 \beta_{11} \mu_{k}^{3}-6 \beta_{16} \mu_{k}^{2}+2\left(2 \beta_{12}+\beta_{66}\right) \mu_{k}-2 \beta_{26}}
$$

It must be borne in mind that for plane strain,

$$
\frac{\partial \beta_{m n}}{\partial a_{p q}}=\delta_{m p} \delta_{n q}-\delta_{m p} \delta_{3 q} \frac{a_{n 3}}{a_{33}}-\delta_{n p} \delta_{3 q} \frac{a_{m 3}}{a_{33}}+\delta_{3 p} \delta_{3 q} \frac{a_{m 3}}{a_{33}} \frac{a_{n 3}}{a_{33}}
$$

while for plane stress,

$$
\frac{\partial \beta_{m n}}{\partial a_{p q}}=\delta_{m p} \delta_{n q}
$$

It is important to point out that the singularity order of the new kernels are equal than in the standard ones. Thus, $\partial T_{i j}(\mathbf{z}, \mathbf{y}) / \partial a_{p q}$ is singular, while $\partial U_{i j}(\mathbf{z}, \mathbf{y}) / \partial a_{p q}$ is weakly (logarithmic) singular, as shown in Appendix B.

\subsection{Discretization of the sensitivity integral equation}

After the discretization process of Equation (12), the sensitivity equation can be written in a simple way

$$
\begin{aligned}
\frac{1}{2} \delta u_{i}(l)+\sum_{k=1}^{N_{e}} \sum_{m=1}^{3} h_{i j}^{m}(l, k) \delta u_{j}^{k}(m)= & \sum_{k=1}^{N_{e}} \sum_{m=1}^{3} g_{i j}^{m}(l, k) \delta t_{j}^{k}(m) \\
& +\sum_{k=1}^{N_{e}} \sum_{m=1}^{3}\left[\delta g_{i j}^{m}(l, k) t_{j}^{k}(m)-\delta h_{i j}^{m}(l, k) u_{j}^{k}(m)\right]
\end{aligned}
$$


where

$$
\begin{aligned}
& \delta h_{i j}^{m}(l, k)=\int_{-1}^{1} \frac{\partial T_{i j}\left(\mathbf{z}(\xi), \mathbf{y}^{l}\right)}{\partial a_{p q}} \phi_{m}(\xi) J^{k}(\xi) \mathrm{d} \xi \\
& \delta g_{i j}^{m}(l, k)=\int_{-1}^{1} \frac{\partial U_{i j}\left(\mathbf{z}(\xi), \mathbf{y}^{l}\right)}{\partial a_{p q}} \phi_{m}(\xi) J^{k}(\xi) \mathrm{d} \xi
\end{aligned}
$$

Computation of the new integration constants $\delta h_{i j}^{m}(l, k)$ and $\delta g_{i j}^{m}(l, k)$ is detailed in Appendix B. Applying Equation (24) at every boundary node, a new system of equations is obtained,

$$
\mathbf{H} \boldsymbol{\delta} \mathbf{u}=\mathbf{G} \boldsymbol{\delta} \mathbf{t}+(\boldsymbol{\delta} \mathbf{G t}-\boldsymbol{\delta} \mathbf{H u})
$$

and after rearranging the columns according to the boundary conditions,

$$
\mathbf{A} \delta \mathbf{x}=\delta \mathrm{Gt}-\delta \mathbf{H u}
$$

whose solution provides the displacement and traction sensitivities at all boundary points.

Note that $\delta u_{i}(\mathbf{y})=0$ at the boundary points where $u_{i}(\mathbf{y})$ is given and, likewise $\delta t_{i}(\mathbf{y})=0$ where $t_{i}(\mathbf{y})$ is known. Therefore, the system matrix $\mathbf{A}$ is the same as for the actual problem.

\subsection{Material sensitivity of strains and stresses at interior and boundary points}

To compute the sensitivity of strains at an interior point, with respect to an arbitrary compliance constant $a_{p q}$, Equation (9) is differentiated with respect to this constant, leading to,

$$
\delta \varepsilon_{i k}(\tilde{\mathbf{z}})=\int_{\Gamma}\left[V_{i j k} \delta t_{j}(\mathbf{z})-S_{i j k} \delta u_{j}(\mathbf{z})\right] \mathrm{d} \Gamma+\int_{\Gamma}\left[\frac{\partial V_{i j k}}{\partial a_{p q}} t_{j}(\mathbf{z})-\frac{\partial S_{i j k}}{\partial a_{p q}} u_{j}(\mathbf{z})\right] \mathrm{d} \Gamma
$$

Two new kernels arise,

$$
\begin{aligned}
\frac{\partial V_{i j k}}{\partial a_{p q}}= & -\sum_{s=1}^{2} \operatorname{Re}\left[\frac{\partial}{\partial a_{p q}}\left\{p_{j s} D_{i k s}\right\} \frac{1}{\left(z_{s}-\tilde{z}_{s}\right)^{2}}+p_{j s} D_{i k s} \frac{\partial}{\partial a_{p q}} \frac{1}{\left(z_{s}-\tilde{z}_{s}\right)^{2}}\right] \\
\frac{\partial S_{i j k}}{\partial a_{p q}}= & \sum_{s=1}^{2} \operatorname{Re}\left[\frac{\partial}{\partial a_{p q}}\left\{q_{j s} D_{i k s}\right\} \frac{\mu_{s} n_{1}-n_{2}}{\left(z_{s}-\tilde{z}_{s}\right)^{2}}+\frac{\partial}{\partial a_{p q}}\left\{\mu_{s} n_{1}-n_{2}\right\} \frac{q_{j s} D_{i k s}}{\left(z_{s}-\tilde{z}_{s}\right)^{2}}\right. \\
& \left.+\frac{\partial}{\partial a_{p q}}\left\{\frac{1}{\left(z_{s}-\tilde{z}_{s}\right)^{2}}\right\} q_{j s} D_{i k s}\left(\mu_{s} n_{1}-n_{2}\right)\right]
\end{aligned}
$$

By a further application of differentiation, and taking into account the derivatives computed in Section 3.1, the new kernels are fully derived.

To obtain the stress tensor, Hooke's law is differentiated with respect to the compliance constant, leading to the final expression,

$$
\delta \sigma_{i}=C_{i j} \delta \varepsilon_{j}-C_{i p} \sigma_{q}
$$


Finally, the sensitivity of strains and stresses on the boundary are computed by a combination of the sensitivities of the longitudinal strain along the boundary and the traction vector components.

\section{APPLICATIONS}

In order to show the accuracy of the proposed approach for computing the material sensitivities, the solutions computed by the numerical BIE-based procedure has been compared to analytical expressions, when available, or finite difference derivatives otherwise.

Several problems have been solved analytically using the complex potential technique [22]. Afterwards, using a symbolic manipulation software, the derivatives of the analytical solution with respect to the material properties have been obtained. The analytical solutions are summarized in Appendix C.

\subsection{Infinite plate with an elliptical cavity under internal loading}

In Figure 1 the geometry and loading of the problem are displayed. The half-axes are such that $a / b=2$. The engineering elastic constants of the plate are: $E_{1}=11.270 \mathrm{GN} / \mathrm{m}^{2}$, $E_{2}=5.880 \mathrm{GN} / \mathrm{m}^{2}, G_{12}=0.686 \mathrm{GN} / \mathrm{m}^{2}, v_{12}=0.076$, therefore the compliance elastic constants are: $a_{11}=0.8503 \times 10^{-1}, a_{16}=0.0, a_{12}=-0.6463 \times 10^{-2}, a_{66}=1.4580, a_{26}=0.0, a_{22}=0.1701$ where the units are $\mathrm{m}^{2} / \mathrm{GN}$. The axes of the ellipse coincide with the orthotropy axes and the cartesian ones. Three loading conditions are considered for the traction angles $\alpha=0,30$ and $45^{\circ}$. Twelve quadratic isoparametric elements model the boundary for the solution by the BEM. Note that the differences between numerical and analytical results are negligible.

In Figures 2 and 3 some of the results are shown. The present approach allows the computation of the sensitivities of displacements, stresses and strains, on the boundary and in the domain, although only partial results are shown here.

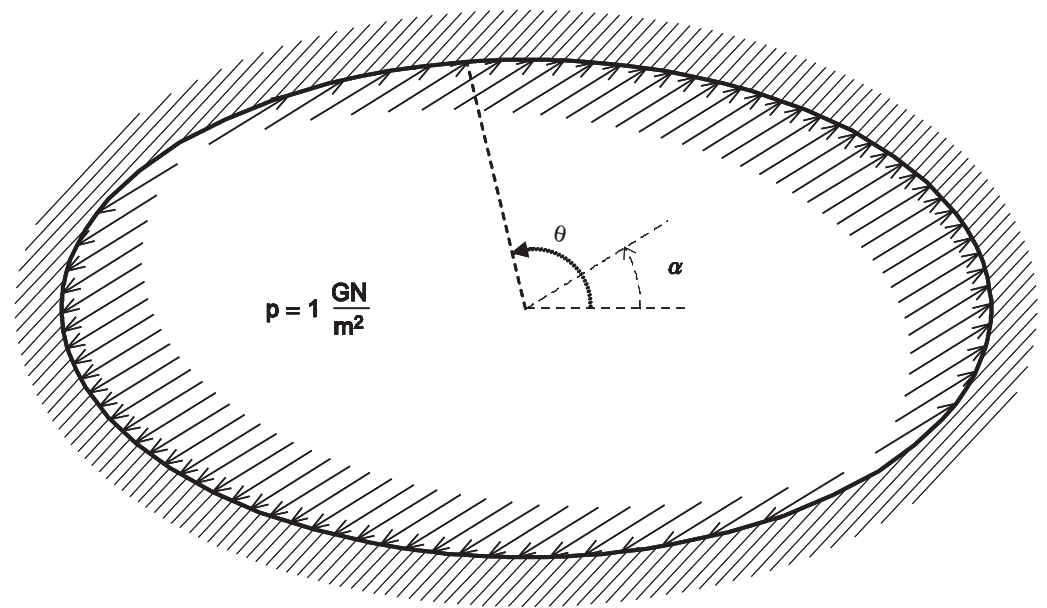

Figure 1. Elliptical cavity in an infinite plate under uniform interior loading. 


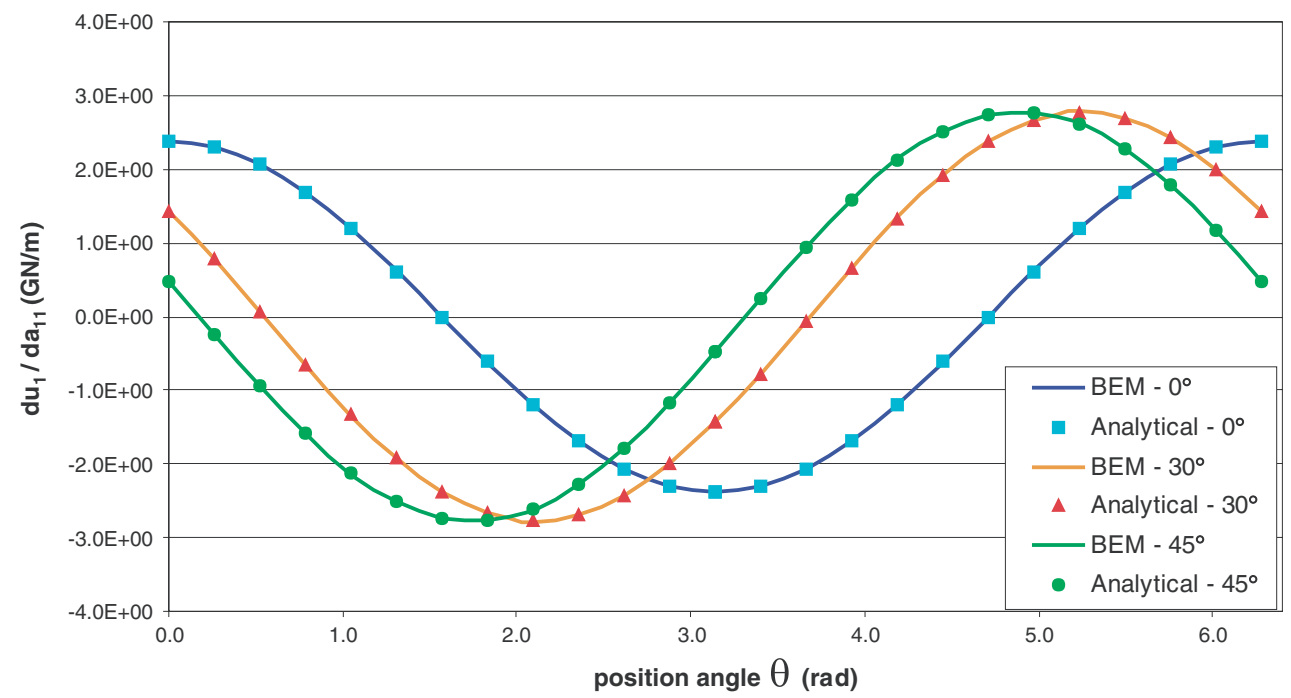

Figure 2. Comparison between the computed numerical value (BEM) and analytical result for the sensitivity of the horizontal displacement along the cavity boundary, with respect to the compliance constants $a_{11}$. Loading inclination 0,30 and $45^{\circ}$.

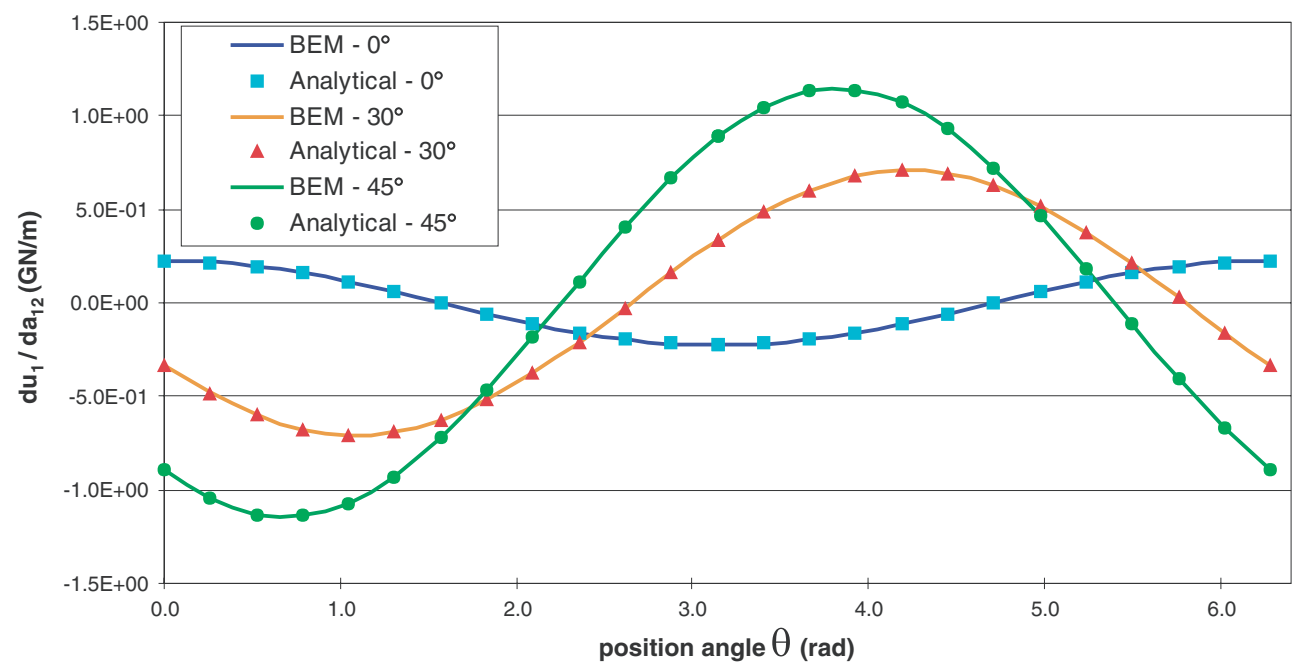

Figure 3. Comparison between the computed numerical value (BEM) and analytical result for the sensitivity of the horizontal displacement along the cavity boundary, with respect to the compliance constants $a_{12}$. Loading inclination 0,30 and $45^{\circ}$.

\subsection{Elliptical core in an infinite plate under remote loading}

In the second application, an elliptical inclusion in an infinite orthotropic plate is considered, as shown in Figure 4. Again the half-axes are such that $a / b=2$. The material of the core is 


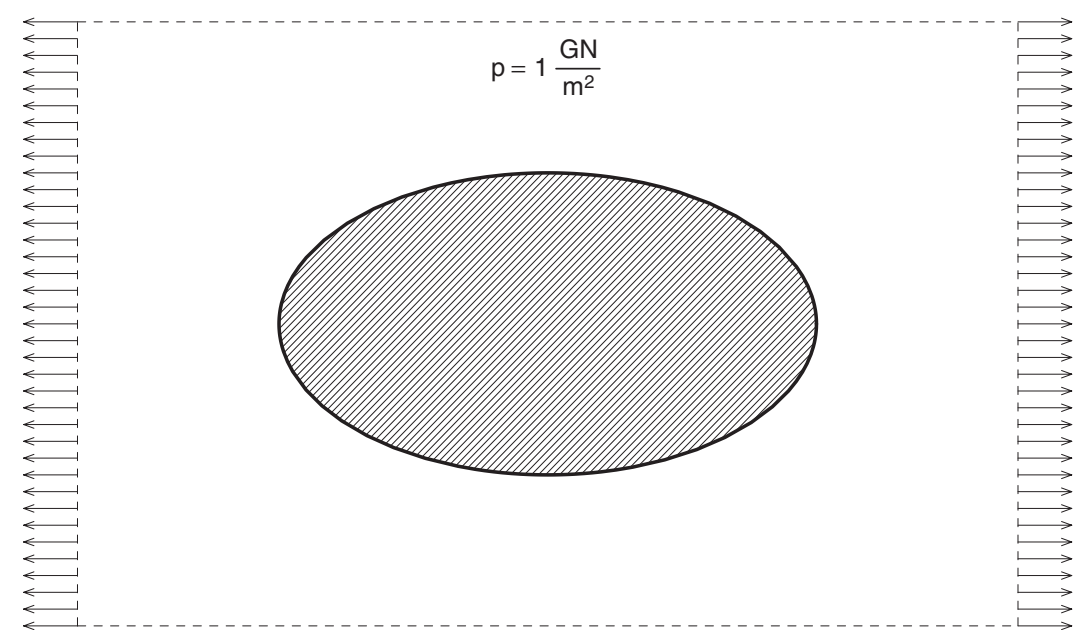

Figure 4. Elliptical orthotropic inclusion in an infinite orthotropic plate, under remote loading.

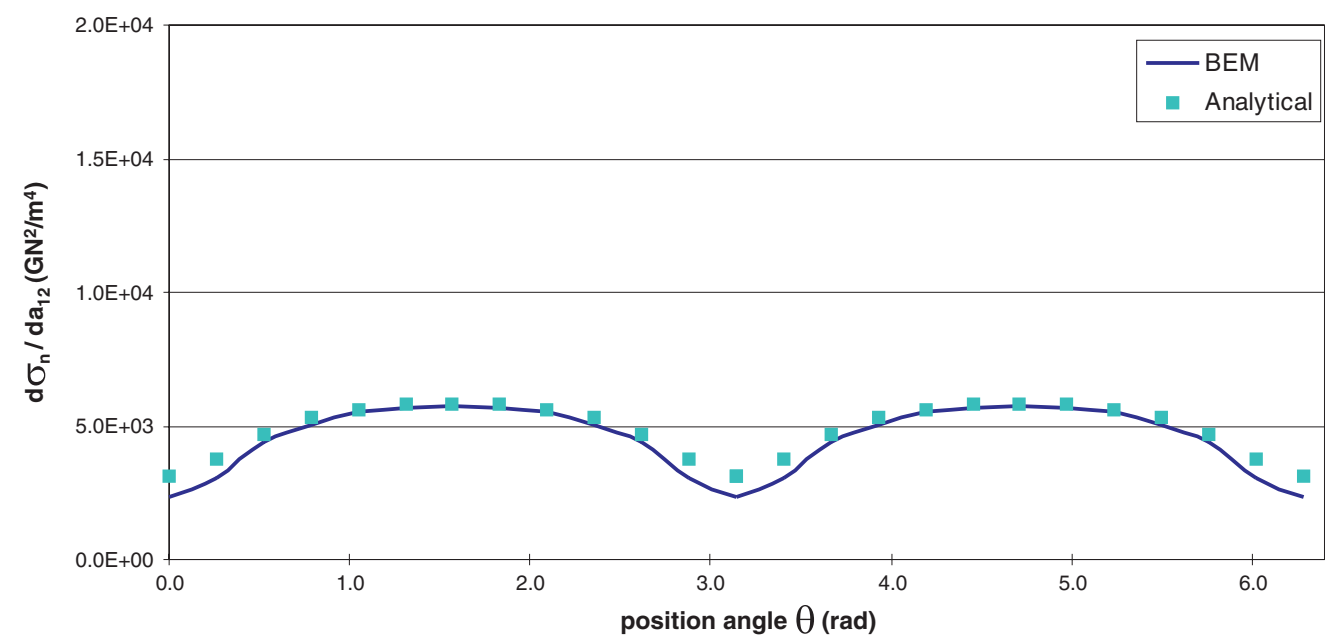

Figure 5. Comparison between the computed numerical value (BEM) and the analytical result for the sensitivity of the normal tension along the matrix-elastic core interface, with respect to the elastic coefficient $a_{12}$.

soldered without negative or positive allowance with the matrix material. The matrix has the same elastic constants as in the previous case, while two cases for the core have been tested. In the first one, the core is orthotropic and its compliance constants are twice those of the matrix. The second one is an absolutely rigid inclusion. Again, 12 elements are employed for the BEM discretization.

In Figures 5-8 the sensitivities of the normal tension on the matrix-core interface with respect to the matrix elastic coefficients $a_{12}$ and $a_{66}$ are shown. 


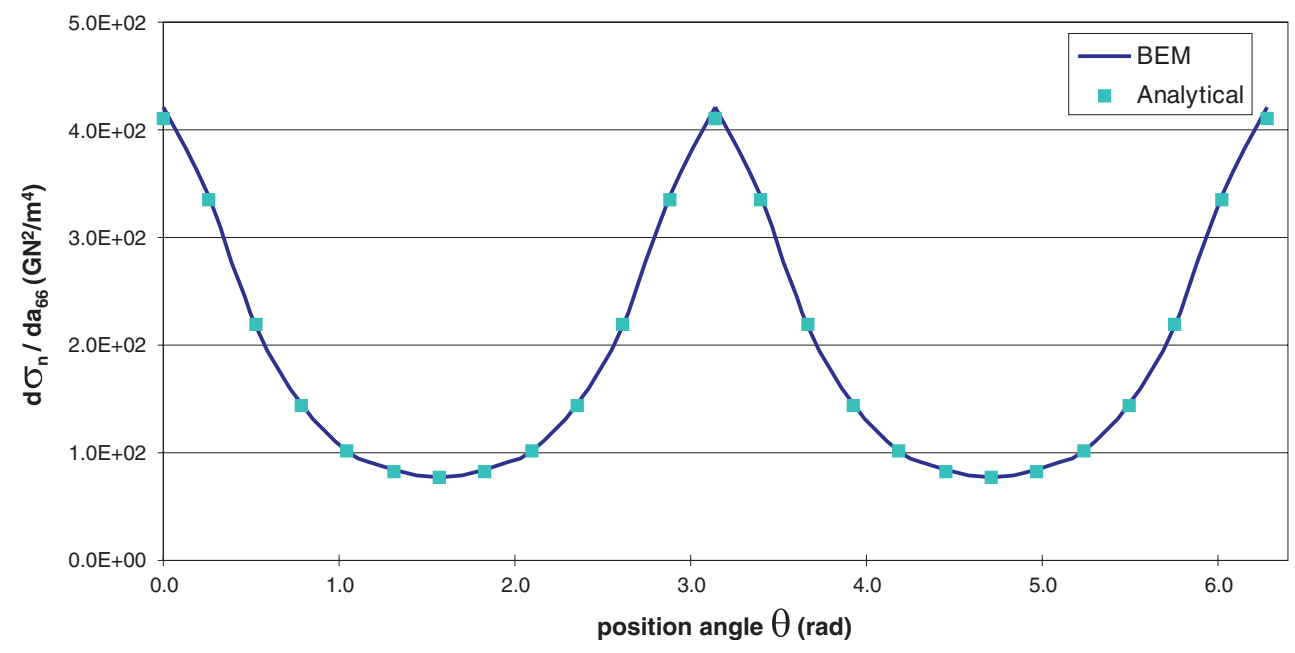

Figure 6. Comparison between the computed numerical value (BEM) and the analytical result for the sensitivity of the normal tension along the matrix-elastic core interface, with respect to the elastic coefficient $a_{66}$.

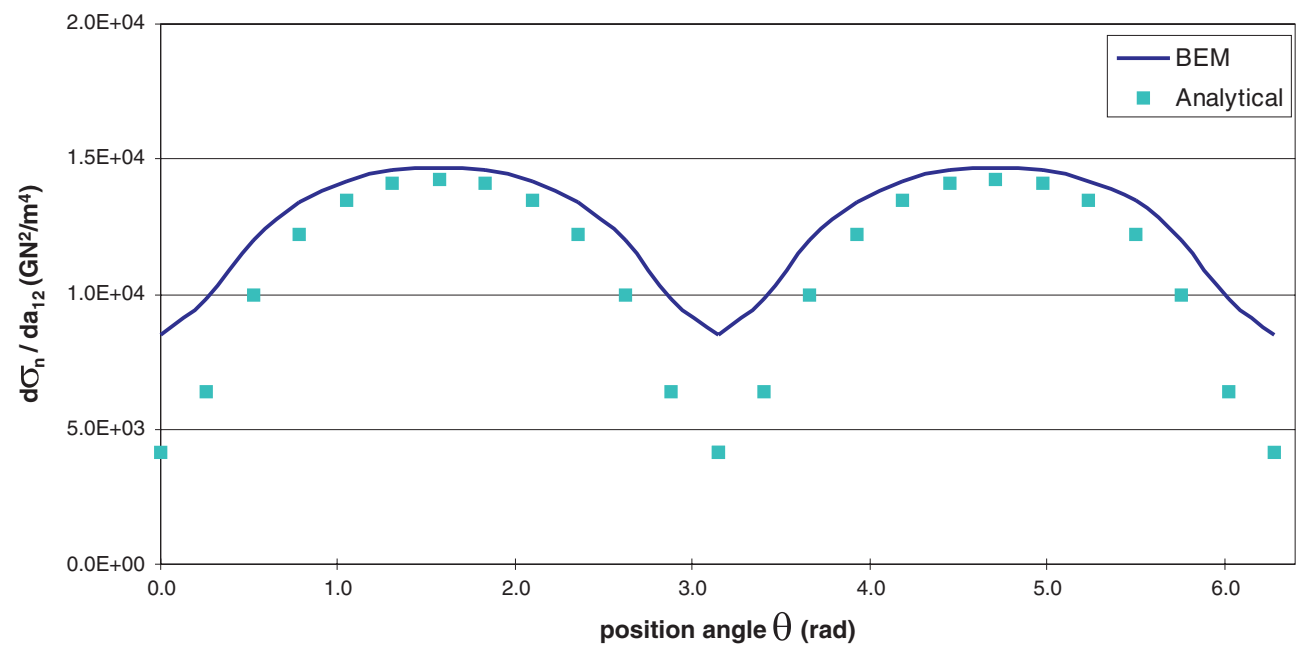

Figure 7. Comparison between the computed numerical value (BEM) and the analytical result for the sensitivity of the normal tension along the matrix-rigid core interface, with respect to the elastic coefficient $a_{12}$.

In this case, there is again a very good match between both solutions for the sensitivities with respect to the constant $a_{66}$, but some discrepancies appear in the sensitivity with respect to $a_{12}$. Actually this is the only case where appreciable differences with respect to the analytical solution have been found. In fact, it might be that the discrepancy is not completely from the 


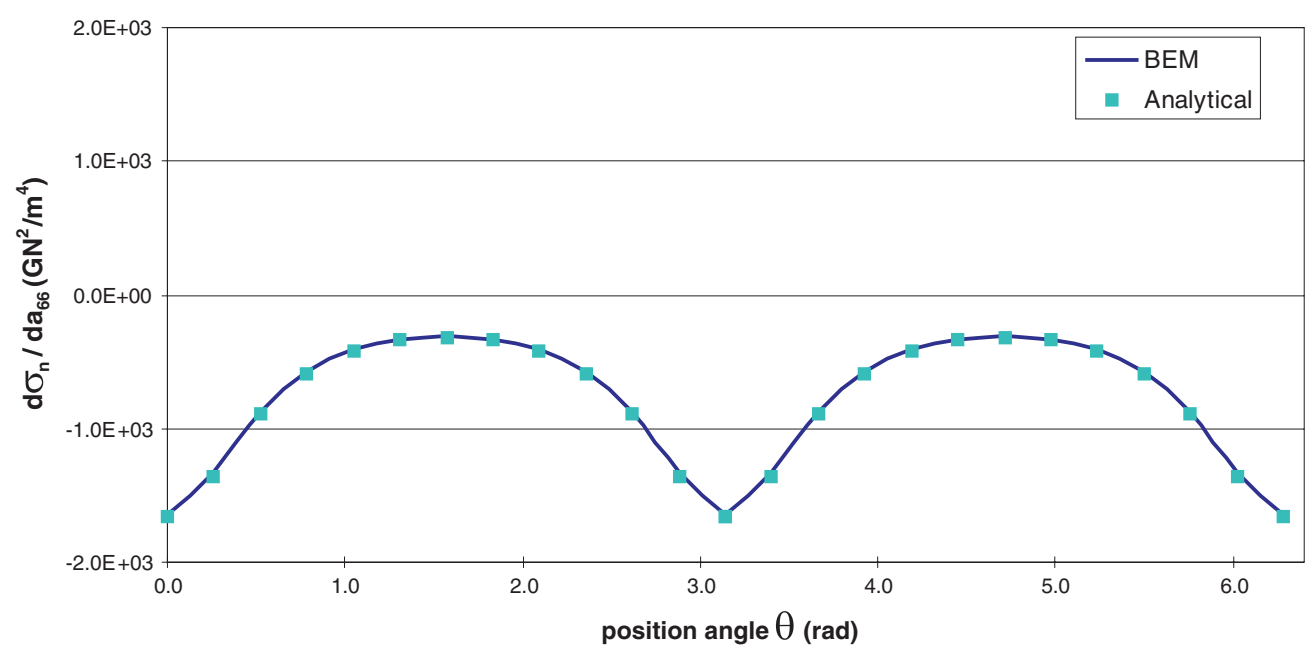

Figure 8. Comparison between the computed numerical value (BEM) and the analytical result for the sensitivity of the normal tension along the matrix-rigid core interface, with respect to the elastic coefficient $a_{66}$.

BEM solution, since the analytical one involves the evaluation of a huge number of terms and some fine-tuning was necessary in establishing the analytical solution for these sensitivities.

Note that, since the domain is piece-wise heterogeneous, a subregional approach have been used [18], and the compliance constants of both regions can be independent variables when computing the sensitivities.

\subsection{Elliptical elastic core in an anisotropic plate}

The last example consists of a finite anisotropic square plate which contains an elliptic inclusion of another anisotropic material. In this case, the matrix material is the same as in previous cases, and the core compliance constants are twice those of the matrix. The ellipse half-axes are such that $a / b=1.5$. Again, the elliptical inclusion is soldered without negative or positive allowance with the matrix. Geometry, loads and boundary conditions are shown in Figure 9. The plate and the inclusion boundaries have been discretized into 24 and 20 isoparametric quadratic elements, respectively. As in the application above sensitivities can be computed with respect to both the inclusion or matrix compliance constants. In order to validate the results obtained with the BEM approach, the results have been compared with the sensitivities computed with finite difference method, since there is no analytical solution available. In this case, the forward difference approach have been used. A finite increment of $1 \%$ of the constant value has been shown to lead to optimal results for finite difference numerical differentiation.

Figures 10-12 show the sensitivity of the horizontal displacements with respect to constants $a_{11}, a_{12}$ and $a_{66}$ of the elastic core. The sensitivities of normal and tangential tractions with respect to the elastic constants $a_{12}$ and $a_{66}$, are displayed in Figures 13-16. All of them show a very good agreement between the sensitivities computed with the BEM approach and the ones calculated using finite differences. 


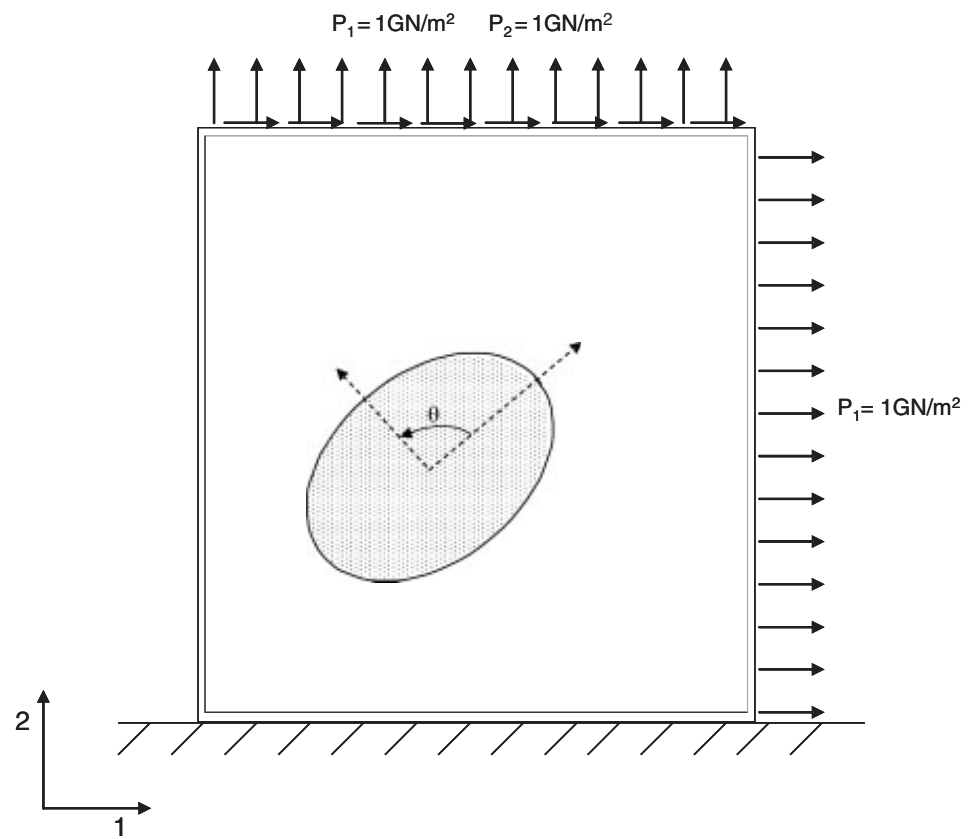

Figure 9. Elliptical orthotropic inclusion in an orthotropic plate.

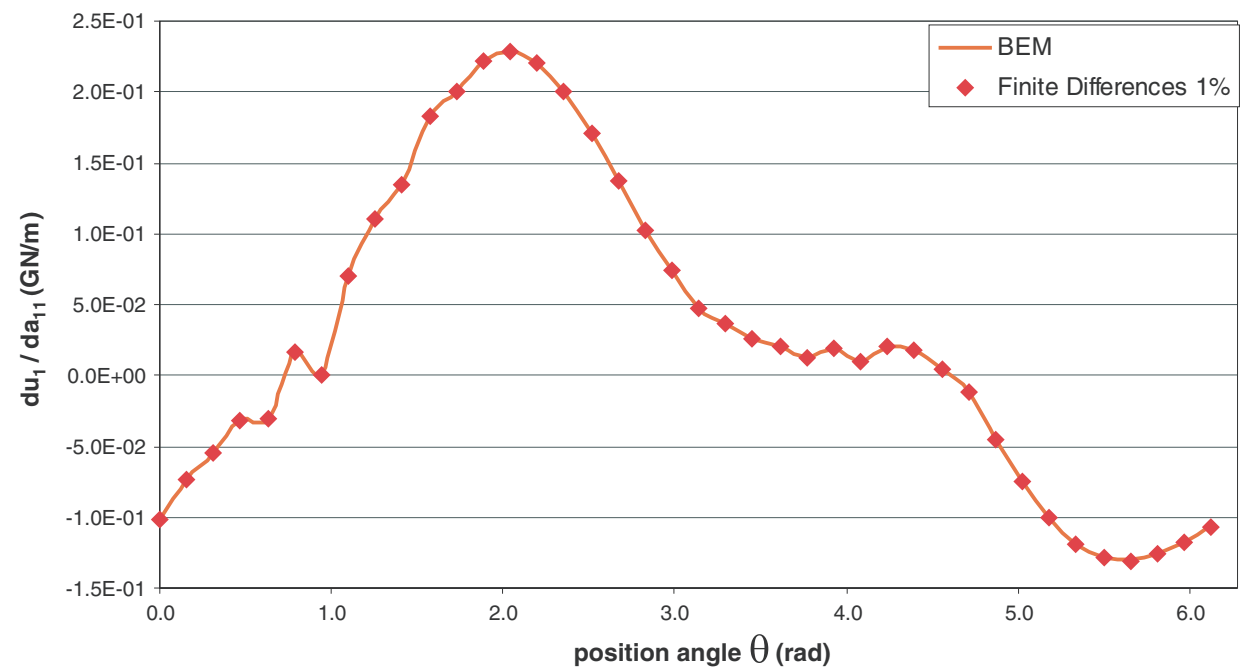

Figure 10. Comparison between the computed numerical values with BEM and with finite difference for the sensitivity of the horizontal displacement along the inclusion boundary, with respect to the compliance constant $a_{11}$. 


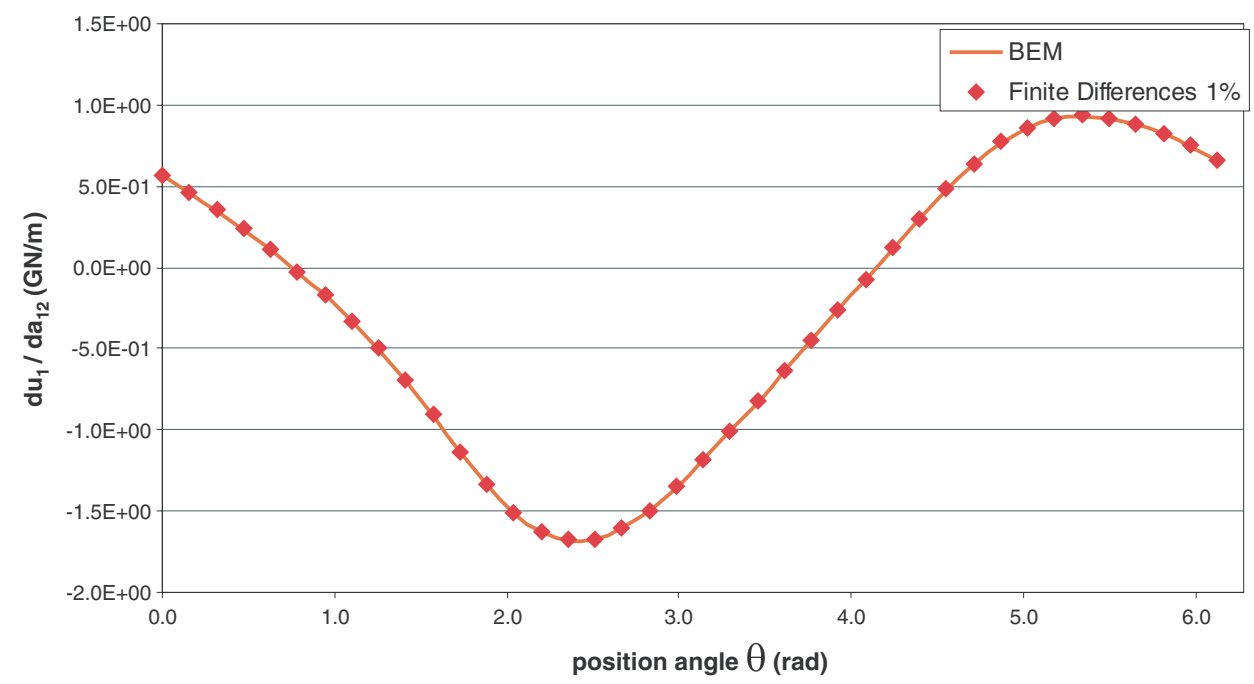

Figure 11. Comparison between the computed numerical values with BEM and with finite difference for the sensitivity of the horizontal displacement along the inclusion boundary, with respect to the compliance constant $a_{12}$.

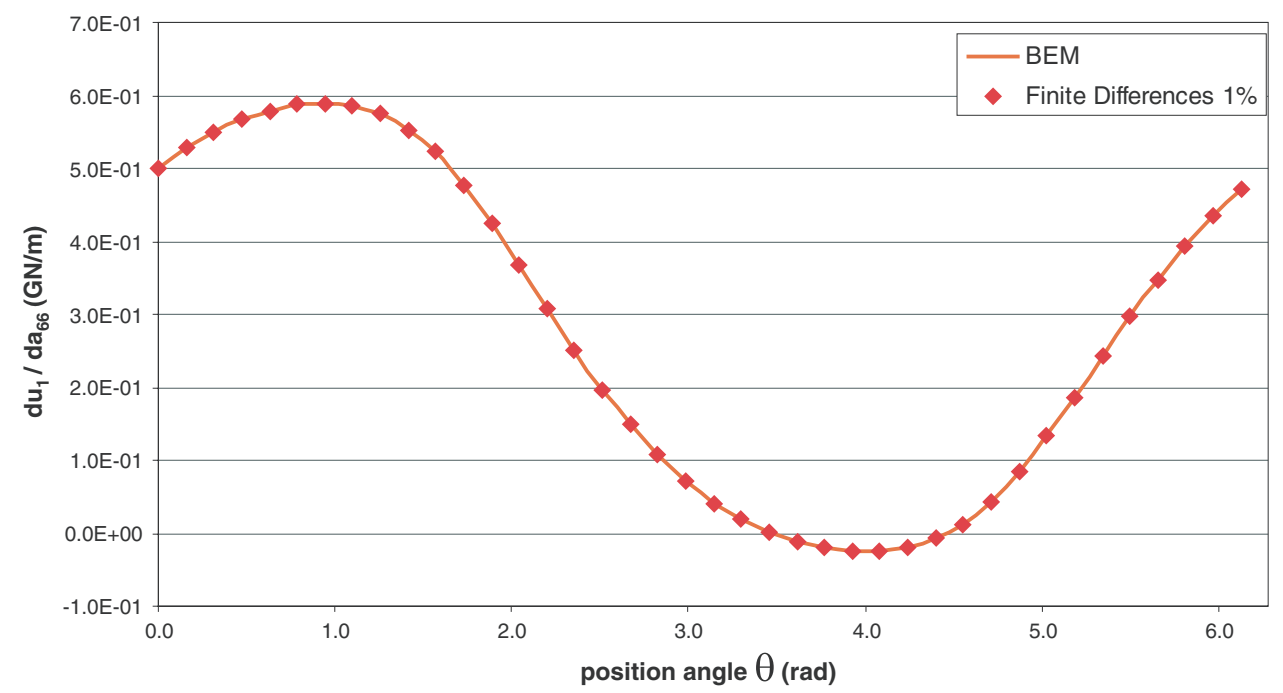

Figure 12. Comparison between the computed numerical values with BEM and with finite difference for the sensitivity of the horizontal displacement along the inclusion boundary, with respect to the compliance constant $a_{66}$. 


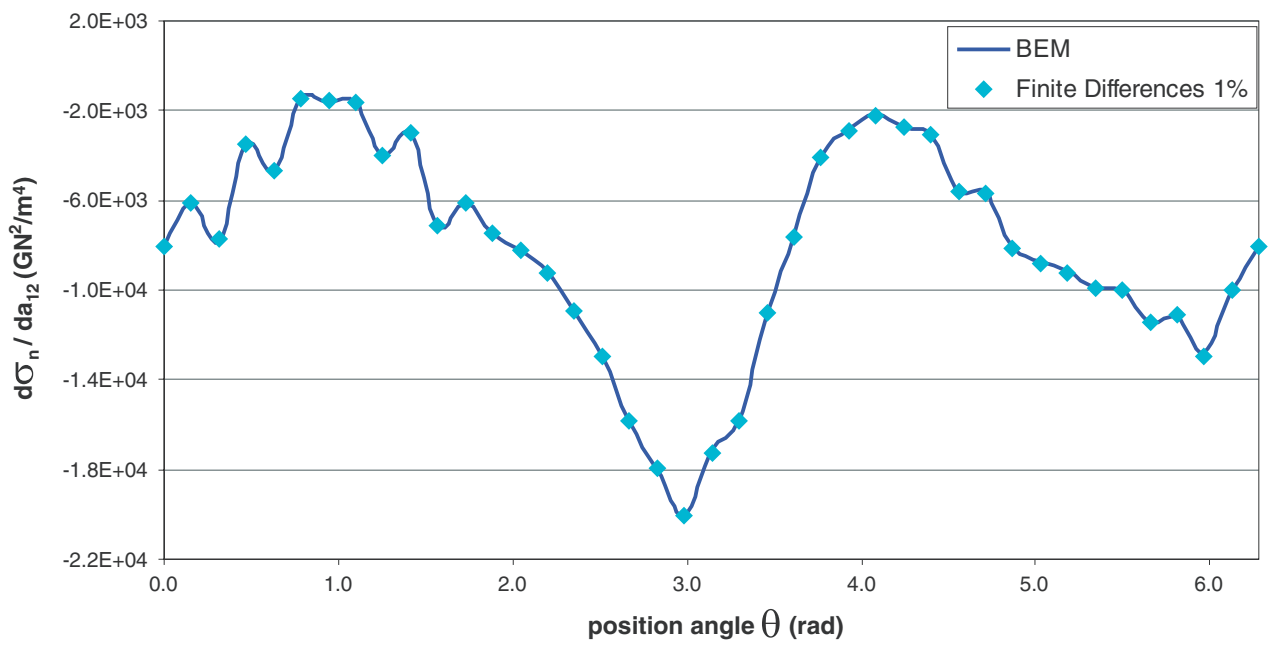

Figure 13. Comparison between the computed numerical values with BEM and with finite difference for the sensitivity of the normal traction along the matrix-elastic core interface, with respect to the elastic coefficient $a_{12}$.

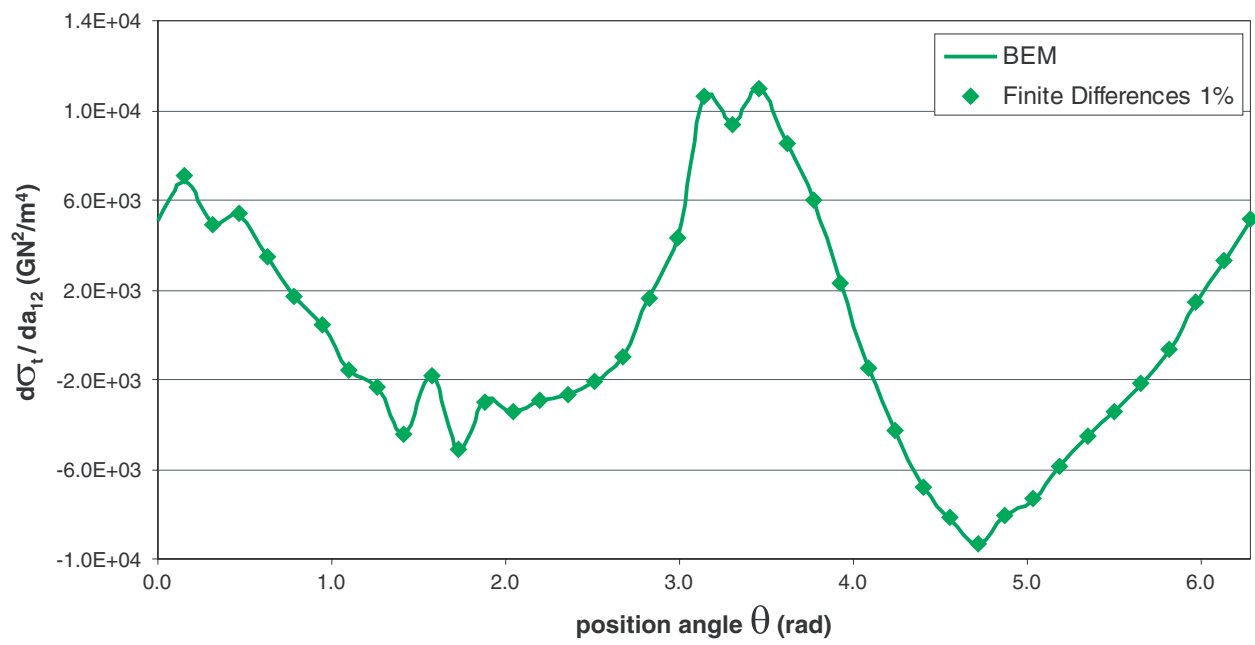

Figure 14. Comparison between the computed numerical values with BEM vs with finite difference for the sensitivity of the tangential traction along the matrix-elastic core interface, with respect to the elastic coefficient $a_{12}$.

\section{CONCLUSIONS}

In this paper an approach based on the BEM for the computation of the sensitivity of elastic fields with respect to the material constants is presented. Two-dimensional rectilinearly anisotropic bodies are considered. 


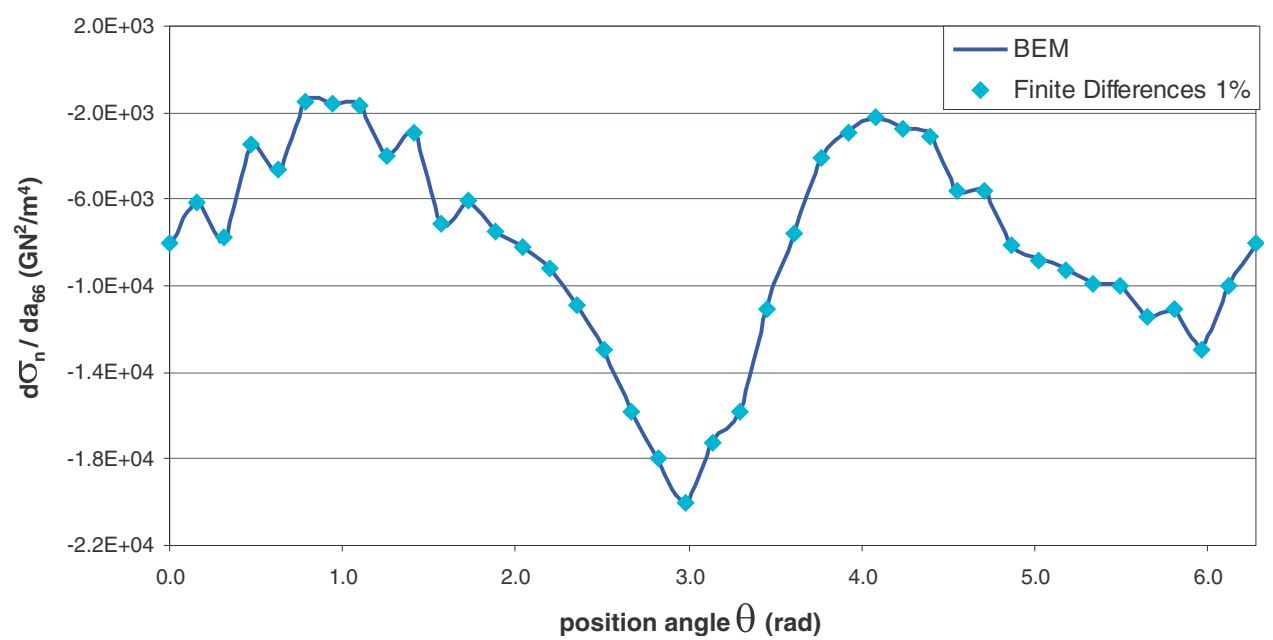

Figure 15. Comparison between the computed numerical values with BEM and with finite difference for the sensitivity of the normal tension along the matrix-elastic core interface, with respect to the elastic coefficient $a_{66}$.

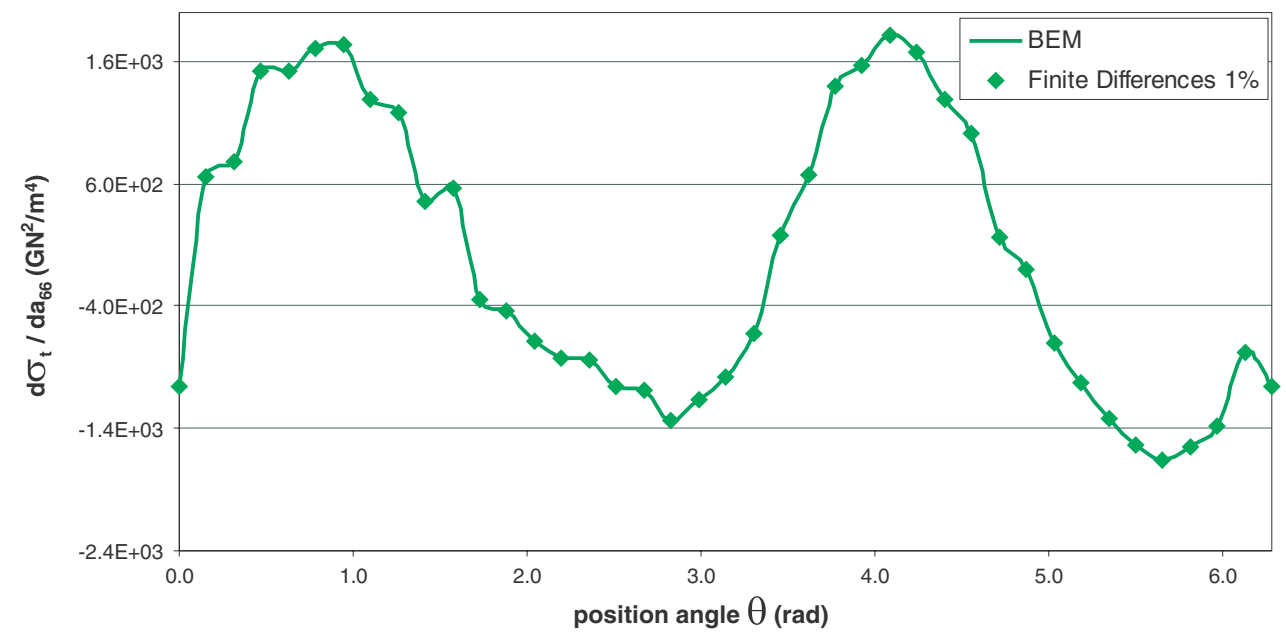

Figure 16. Comparison between the computed numerical values with BEM and with finite difference for the sensitivity of the tangential tension along the matrix-elastic core interface, with respect to the elastic coefficient $a_{66}$.

The sensitivity BIE has been obtained and an approach based on standard BEM techniques is proposed for its numerical solution. It is shown that the new kernels that arise in the sensitivity BIE are as singular as the standard kernels. Their integration has been performed by an analytical-numerical decomposition, which yields very accurate results. 
The accuracy of this numerical approach is contrasted to solutions obtained analytically by the complex potential theory, when available, and the results prove that the proposed approach is highly reliable, even using coarse meshes. In the last application, where no analytical solution can be found, the sensitivities computed by BEM are compared to finite difference results: again, there is a very close agreement between both solutions.

The implementation allows the computation of sensitivities for piece-wise heterogeneous bodies with respect to the material properties of any subdomain.

The computed sensitivities can be employed to implement an inverse procedure for computing the material constant of a body or part of it (inclusions, coating) by minimizing a suitable functional. Likewise, they can be employed in estimating the reliability of a given solution and computing its statistical parameters from those of the material constant distributions.

\section{APPENDIX A: EVALUATION OF THE INTEGRATION CONSTANTS}

Two cases must be distinguished during the evaluation of the integration constants. First, the ordinary case, when the collocation point does not belong to the element where the expression is evaluated. This one does not present any particular difficulty and the integrals can be computed using Gaussian quadrature. On the contrary, the singular case, i.e. when the collocation point is within the evaluation element, needs some detailed development.

Computation of $h_{i j}^{m}(l, k)$ can be expressed in the form

$$
h_{i j}^{m}(l, k)=2 \mathbb{R e}\left[\int_{-1}^{1} \sum_{s=1}^{2} \frac{q_{j s} A_{i s}}{z_{s}(\xi)-y_{s}^{l}}\left(\mu_{s} n_{1}-n_{2}\right) \phi_{m} J^{k} \mathrm{~d} \xi\right]
$$

which implies evaluation of the complex integral

$$
I_{s}^{m}(l, k)=\int_{-1}^{1} \frac{\mu_{s} n_{1}-n_{2}}{z_{s}(\xi)-y_{s}^{l}} \phi_{m} J^{k} \mathrm{~d} \xi
$$

When $l \in k$ (singular case), $I_{s}^{m}(l, k)$ can be separated into a regular integral $\tilde{I}_{s}^{m}(l, k)$ plus and a VPC as follows:

$$
I_{s}^{m}(l, k)=\tilde{I}_{s}^{m}(l, k)+f_{-1}^{1} \frac{\phi_{m}}{\xi-\xi_{l}} \mathrm{~d} \xi
$$

where $\xi_{l}$ is the natural co-ordinate of the collocation point. The second integral is solved taking into account that the two infinite terms which appear when $\xi_{l}=1$ or -1 , cancel out when the evaluation is undertaken in two adjacent elements.

$$
f_{-1}^{1} \frac{\phi_{m}}{\xi-\xi_{l}} \mathrm{~d} \xi=f_{-1}^{1} \frac{a \xi^{2}+b \xi+c}{\xi-\xi_{l}} \mathrm{~d} \xi=2\left(a+b \xi_{l}\right)+\phi_{m}\left(\xi_{l}\right) \ln \frac{1-\xi_{l}}{1+\xi_{l}}
$$

On the other hand, the $g_{i j}^{m}(l, k)$ constants are expressed in the following form:

$$
g_{i j}^{m}(l, k)=2 \mathbb{R e}\left[\int_{-1}^{1} \sum_{s=1}^{2} p_{j s} A_{i s} \ln \left[z_{s}(\xi)-y_{s}^{l}\right] \phi_{m} J^{k} \mathrm{~d} \xi\right]
$$


a formula which includes the complex expression

$$
I_{s}^{m}(l, k)=\int_{-1}^{1} \ln \left[z_{s}(\xi)-y_{s}^{l}\right] \phi_{m} J^{k} \mathrm{~d} \xi
$$

In the singular case this integral becomes improper, but after some manipulations can be cast in the form,

$$
\int_{0}^{1} \ln \left(\frac{1}{\eta}\right) f(\eta) \mathrm{d} \eta
$$

which is computed using specific Gaussian quadrature with logarithmic weight [18].

\section{APPENDIX B: EVALUATION OF THE SENSITIVITY EQUATION INTEGRATION CONSTANTS}

As usual, two distinct cases appear for evaluating the constant $\delta h_{i j}^{m}(l, k), \delta g_{i j}^{m}(l, k)$. The simpler one arises when the collocation point does not belong to the integration element. In this case, standard Gaussian quadrature is employed, although the number of abscissas will depend on the distance between the node and the element.

In the second case, the node belongs to the element, the new expressions which appear when computing $\delta h_{i j}^{m}(l, k)(25)$ involve two kinds of integrals. Firstly, a singular integral as that given in Equation (A2), whose integration is shown in Appendix A. Secondly, an integral in the form

$$
I_{s}^{m}(l, k)=\int_{-1}^{1}\left[\frac{x_{2}(\xi)-x_{2}^{l}}{\left(z_{s}(\xi)-y_{s}^{l}\right)^{2}}\left(\mu_{s} n_{1}-n_{2}\right)-\frac{n_{1}}{z_{s}(\xi)-y_{s}^{l}}\right] \phi_{m} J^{k} \mathrm{~d} \xi
$$

which seems to be singular or even hypersingular. However, if the integrand in $I_{s}^{m}(l, k)$ is expanded, it is easily proved that all the singular terms cancel out.

When computing the constant $\delta g_{i j}^{m}(l, k)(26)$, two kinds of integrals appear. First, a logarithmic one, which is numerically computed as in Appendix A, and secondly, integrals in the form

$$
I_{s}^{m}(l, k)=\int_{-1}^{1} \frac{x_{2}(\xi)-x_{2}^{l}}{z_{s}(\xi)-y_{s}^{l}} \phi_{m} J^{k} \mathrm{~d} \xi
$$

which are computed using a standard Gaussian quadrature, since they are regular.

\section{APPENDIX C: ANALYTICAL MATERIAL SENSITIVITY FOR CAVITIES AND INCLUSIONS IN AN INFINITE PLANE}

The first case treated is an anisotropic infinite plate, with the loads and geometry establishing a plane stress state. We consider an elliptic cavity inside, the boundary of which is 
subjected to an uniform internal traction, forming an angle $\alpha$ with the horizontal axis (see Figure 1).

Below, the analytical expressions for the normal stress, $\sigma_{n}$, and horizontal displacement, $u_{1}$, along the boundary of the cavity, are detailed. The angle $\theta$ is the position angle and $\alpha$ is the inclination of the uniform load, $P$, both referenced to the horizontal axis. In addition, $a$ and $b$ are the horizontal and vertical semi-axes of the ellipse, respectively.

$$
\begin{aligned}
\frac{\sigma_{n}}{P}= & \mathbb{R e}\left[\frac { B } { A C } \left\{b ^ { 2 } \operatorname { c o s } ^ { 2 } \theta \left(a \mu_{1} \mu_{2} \sin \alpha \sin \theta\right.\right.\right. \\
& \left.-\cos \alpha\left(b \mu_{1} \mu_{2} \cos \theta-a\left(\mu_{1}+\mu_{2}\right) \sin \theta\right)\right) \\
& -2 a b \sin \theta \cos \theta\left(b \mu_{1} \mu_{2} \sin \alpha \cos \theta+a \cos \alpha \sin \theta\right) \\
& \left.\left.+a^{2} \sin ^{2} \theta\left(b \cos \alpha \cos \theta+\sin \alpha\left(b\left(\mu_{1}+\mu_{2}\right) \cos \theta-a \sin \theta\right)\right)\right\}\right] \\
\frac{u_{1}}{P}= & -\llbracket \mathrm{m}\left[B\left(\cos \alpha\left(a_{16}-a_{11}\left(\mu_{1}+\mu_{2}\right)\right)+\sin \alpha\left(a_{12}-a_{11} \mu_{1} \mu_{2}\right)\right)\right]
\end{aligned}
$$

where

$$
\begin{aligned}
& A=b^{2} \cos ^{2} \theta+a^{2} \sin ^{2} \theta \\
& B=(b \cos \alpha+\imath a \sin \alpha)(\cos \theta-\imath \sin \theta) \\
& C=\left(b \mu_{1} \cos \theta-a \sin \theta\right)\left(b \mu_{2} \cos \theta-a \sin \theta\right)
\end{aligned}
$$

The second case studied is an elliptic core inserted in a plate. The core is soldered without negative allowance. Two possibilities are considered, one with an elastic core whose compliance constants are twice those of the matrix. The second is an absolutely rigid inclusion. In both cases, the plate is under a remote horizontal loading $P$. Since the complete expressions are very unwieldy, only the normal stresses on the boundary of the inclusion are shown, and then only for a particular case, namely when matrix and core are orthotropic, material axes are parallel to the cartesian ones, and the horizontal and vertical semi-axes of the ellipse are $a=2$ and $b=1$.

In the elastic core case, normal stresses along the interface are,

$$
\begin{aligned}
\frac{\sigma_{n}}{P}= & \frac{1}{\cos ^{2} \theta+4 \sin ^{2} \theta}\left\{\cos ^{2} \theta\left(1+2 \mathbb{R e}\left[\frac{\left(I+\mu_{1} J\right) \mu_{2}^{2}}{K}-\frac{\left(I+\mu_{2} J\right) \mu_{1}^{2}}{L}\right]\right)\right. \\
& -8 \sin \theta \cos \theta \mathbb{R e}\left[\frac{\left(I+\mu_{1} J\right) \mu_{2}}{K}-\frac{\left(I+\mu_{2} J\right) \mu_{1}}{L}\right] \\
& \left.+8 \sin ^{2} \theta \mathbb{R e}\left[\frac{I+\mu_{1} J}{K}-\frac{I+\mu_{2} J}{L}\right]\right\}
\end{aligned}
$$


and for the rigid one,

$$
\begin{aligned}
\frac{\sigma_{n}}{P}= & \frac{1}{\cos ^{2} \theta+4 \sin ^{2} \theta}\left\{\cos ^{2} \theta\left(1+2 \mathbb{R e}\left[\frac{\left(Q+\mu_{1} R\right) \mu_{2}^{2}}{S}-\frac{\left(Q+\mu_{2} R\right) \mu_{1}^{2}}{T}\right]\right)\right. \\
& -8 \sin \theta \cos \theta \mathbb{R e}\left[\frac{\left(Q+\mu_{1} R\right) \mu_{2}}{S}-\frac{\left(Q+\mu_{2} R\right) \mu_{1}}{T}\right] \\
& \left.+8 \sin ^{2} \theta \mathbb{R e}\left[\frac{Q+\mu_{1} R}{S}-\frac{Q+\mu_{2} R}{T}\right]\right\}
\end{aligned}
$$

where

$$
\begin{aligned}
& A=\sqrt{\frac{2 a_{12}}{a_{11}}+2 \sqrt{\frac{a_{22}}{a_{11}}}+\frac{a_{66}}{a_{11}}}, \quad B=\frac{2 a_{12}}{a_{11}}+\sqrt{\frac{a_{22}}{a_{11}}}, \quad C=\frac{a_{12}}{\sqrt{a_{22} a_{11}}} \\
& D=\frac{1}{a_{66}}\left(2\left(5 a_{12} C+4 a_{11} \sqrt{\frac{a_{22}}{a_{11}}}+a_{12}(1-4 C)+a_{22}\left(-2 a_{12}+4 a_{11} A\right)\right)\right) \\
& E=2+\frac{2}{a_{66}}\left(a_{12}+a_{11} B+\sqrt{a_{11} a_{22}} A\right), \quad F=\frac{2}{a_{66}} \sqrt{\frac{a_{22}}{a_{11}}}(2+A) \\
& G=(\cos \theta+\imath \sin \theta)^{2}, \quad H=-2\left(\frac{a_{12}}{a_{11}}+\sqrt{\frac{a_{22}}{a_{11}}}\right) \\
& I=1-\frac{2 a_{12}+2 a_{66}+4 A a_{11}+2 B a_{11}+F a_{11} a_{66}}{a_{66}(D+E)} \\
& J=\frac{2 \imath(4+A-(2+A)(1-C))}{-2 \sqrt{\left(\frac{a_{22}}{a_{11}}-2 A\right)(4+A)+(1-C) H}} \\
& K=\left(\mu_{1}-\mu_{2}\right)\left(2 l(G-1)+(G+1) \mu_{2}\right), \quad L=\left(\mu_{1}-\mu_{2}\right)\left(2{ }_{l}(G-1)+(G+1) \mu_{1}\right) \\
& M=\frac{2 a_{12}}{a_{66}}(1+C), \quad N=\frac{2}{a_{66}}\left(a_{66}-a_{12}+a_{11} B\right), \quad O=2\left(\frac{a_{12}}{a_{11}}-\sqrt{\frac{a_{22}}{a_{11}}}\right) \\
& Q=1-\frac{2}{a_{66}(M+N)}\left(a_{12}+a_{66}+a_{11}(A+B)\right) \\
& R=\frac{2 l(A-(2+A)(1+C))}{2 A^{2}+(1+C) O} \\
& S=\frac{1}{a_{66}}\left(\mu_{1}-\mu_{2}\right)\left(2 \imath\left(a_{66}-1\right)+\left(a_{66}+1\right) \mu_{2}\right) \\
& T=\frac{1}{a_{66}}\left(\mu_{1}-\mu_{2}\right)\left(2 l\left(a_{66}-1\right)+\left(a_{66}+1\right) \mu_{1}\right)
\end{aligned}
$$


To obtain the stress and displacement material sensitivities, these expressions are differentiated with respect to the compliance constants. The resultant expressions are not shown due to being rather unwieldy.

\section{REFERENCES}

1. Wang K. Vibration analysis of cracked composite bending-torsion beams for damage diagnosis. Ph.D. Thesis, Virginia Polytechnic Institute, 2004.

2. Hamey CS, Lestari W, Qiao P, Song G. Experimental damage identification of carbon/epoxy composite beams using curvature mode shapes. Structural Health Monitoring 2004; 3(4):333-353.

3. Doyle JF. Modern Experimental Stress Analysis: Completing the Solution of Partially Specified Problems. Wiley: New York, 2004.

4. Bui HD. Inverse Problems in the Mechanics of Materials. An Introduction. CRC Press: Eyrolles, 1994.

5. Kubo S. Classification of inverse problems arising in field problems and their treatments. In Inverse Problems in Engineering Mechanics, Bui HD, Tanaka M (eds). Springer: Tokyo, Japan, 1992; 51-60.

6. Schnur D, Zabaras N. An inverse method for determining elastic material properties and a material interface. International Journal for Numerical Methods in Engineering 1992; 33:2039-2057.

7. Hasan WM, Piva A, Viola E. Parameter estimation from measured displacements on crack edges in an isotropic material. Engineering Fracture Mechanics 1998; 59:697-712.

8. Constantinescu A, Tardieu N. Identification strategies for recovering material parameters from indentation experiments. In Inverse Problems in Engineering Mechanics II, Tanaka M, Dulikravich G (eds). Elsevier: Nagano, Japan, 2000.

9. Tadi M. Evaluation of elastic properties in 2-d non-homogeneous solids. Computational Mechanics 2001; 27:208-217.

10. Xiang Z, Swoboda G, Cen Z. Identification of damage parameters for jointed rock. Computers and Structures 2002; 80:1429-1440.

11. Marin L, Elliot L, Ingham DB, Lesnic D. Parameter identification in isotropic linear elasticity using the boundary element method. Engineering Analysis with Boundary Elements 2003; 28:221-233.

12. Frederiksen PS. Parameter uncertainty and the design of optimal experiments for the estimation of elastic constant. International Journal of Solids and Structures 1998; 35:1241-1260.

13. Wang WT, Kam TY. Elastic constants identification of shear deformable laminated composite plates. Journal of Engineering Mechanics 2001; 127(11):1117-1123.

14. Cailis G, Rikards R, Chate A. Identification of elastic properties of laminates based on experimental design. International Journal of Solids and Structures 2001; 38:5097-5115.

15. Lauwagie T, Sol H, Roebben G, Heylen W, Shi Y, van der Biest O. Mixed numerical-experimental identification of elastic properties of orthotropic metal plates. NDT\&E International 2003; 36:487-495.

16. Huang L, Sun X, Liu Y, Cen Z. Parameter identification for two-dimensional orthotropic material bodies by the boundary element method. Engineering Analysis with Boundary Elements 2003; 28(2):109-121.

17. Heyliger P, Ugander P, Ledbetter H. Anisotropic elastic constants: measurement by impact resonance. Journal of Materials in Civil Engineering 2001; 13(5):356-363.

18. Brebbia CA, Domínguez J. Boundary Elements, An Introductory Course. WIT Press, Computational Mechanics Publications: Southampton, 1992.

19. Sollero P. Fracture mechanics analysis of anisotropic laminates by the boundary element method. Ph.D. Thesis, Wessex Institute of Technology, 1994.

20. Aliabadi MH. The Boundary Element Method: Applications in Solids and Structures, vol. 2. Wiley: New York, 2002.

21. Schlar NA. Anisotropy Analysis Using Boundary Elements. Topics in Engineering, vol. 20. Computational Mechanics Publications: Southampton, 1994.

22. Lekhnitskii SG. Theory of Elasticity of an Anisotropic Body. MIR Publishers: Moscow, 1981. 